\title{
Complicaciones sistémicas derivadas de celulitis facial odontogénica en niños Venezolanos menores de 6 años.
}

Gabriel Alejandro Zambrano ${ }^{1}$;

Rosa Gabriela Rondón ";

María Elena Guerra ${ }^{3}$

\section{Resumen}

Objetivo: Determinar la prevalencia de complicaciones sistémicas derivadas de celulitis facial odontogénica y su relación con el peso y la talla de niños de 1 a 6 años hospitalizados en el Servicio de Infectología Pediátrica del Hospital Universitario de Caracas en el periodo 20062014. Materiales y métodos: Se realizó un estudio epidemiológico de prevalencia, no experimental, retrospectivo, transversal y analítico en una muestra seleccionada a conveniencia de 310 niños en edades entre 1 y 6 años, con análisis de frecuencias, porcentajes, promedios, prueba de significancia estadística chi cuadrado, correlación de Pearson y Odds-Ratio. Resultados: la prevalencia de complicaciones sistémicas derivadas de la celulitis facial odontogénica en esta muestra fue del 32\%, y se reportan 5: meningitis $15 \%$, absceso cerebral $2 \%$, neumonía $6 \%$, endocarditis infecciosa $5 \%$ y septicemia $4 \%$. El $65,48 \%$ y el $68,06 \%$ de la muestra total pre- sentó talla baja y peso bajo respectivamente. El peso bajo se relacionó estadísticamente con la presencia de complicaciones sistémicas en esta población. Al comparar con niños de talla y peso normal, los niños con baja talla (percentil 10) tuvieron una probabilidad 3 veces mayor de presentar complicaciones y los de bajo peso (percentil 10) presentaron 4 veces más probabilidades de presentar complicaciones sistémicas. Conclusiones: las complicaciones sistémicas que se pueden derivar de una celulitis facial odontogénica son varias y pueden comprometer la vida del paciente, en este estudio la más frecuente fue la meningitis. El peso y la talla son variables importantes a considerar al evaluar y tratar enfermedades infecciosas odontogénicas en la población pediátrica ya que pueden ser factores de riesgo en la aparición de complicaciones sistémicas asociadas.

Palabras clave: celulitis facial odontogénica, complicaciones sistémicas, bajo peso, baja talla

\footnotetext{
1. Especialista en Odontología Infantil. MSc en Odontología. Profesor Instructor Centro de atención a pacientes con enfermedades infectocontagiosas CAPEI-UCV. Doctorando en el Programa de Doctorado en Odontología, Universidad Central de Venezuela, Caracas, Venezuela.

2. Odontólogo, Especialista en Ortodoncia, Residente de la Maestría Individualizada en Odontología. Facultad de Odontología. Universidad Central de Venezuela, Caracas, Venezuela.

3. Especialista en Odontología Infantil. Doctora en Ciencias mención salud materno-infantil. Profesor Titular Centro de atención a pacientes con enfermedades infectocontagiosas CAPEI-UCV. Facultad de Odontología. Universidad Central de Venezuela, Caracas, Venezuela.
} 


\section{Complicações sistêmicas de celulite facial odontogenic em crianças venezuelanas menores de 6 anos.}

\section{Resumo}

Determinar a prevalência de complicações sistêmicas de celulite facial odontogenica e sua relação com o peso e altura de crianças de 1 a 6 anos hospitalizadas nas Serviço de Doenças Infecciosas Pediátricas del Hospital Universitário de Caracas, no período 2006-2014. Materiais e métodos. Um estudo epidemiológico de prevalência foi realizado, não-experimental, retrospectivo, transversal e analíticas em uma amostra de conveniência selecionada de 310 crianças de 1 a 6 anos, com análise de freqüência, porcentagens, médias, teste de shows de significância estatística chi quadrado, de correlação de Pearson e Odds ratio. Resultados. A prevalência de complicações sistémicas de celulite facial odontogénica nesta amostra foi de $32 \%$, e relatou 5 : $15 \%$ meningite, abcesso cerebral $2 \%, 6 \%$ pneumonia, endocardite infecciosa e septicemia 5\% 4\%. O $65,48 \%$ e ele $68,06 \%$ e a amostra total mostrou LHA e peso respectivamente. Baixo peso foi estatistica- mente relacionada com a presença de complicações sistêmicas nesta população. Quando comparado com crianças de estatura normal e peso, crianças com baixa estatura (percentil 10) tinha 3 vezes mais propensos a ter complicações e baixo peso ao nascer (percentil 10) apresentou 4 vezes mais propensos a ter complicações Conclusões. Complicações sistémica que pode ser derivada a partir de celulite facial odontogénica são vários e podem comprometer a vida do paciente, no presente estudo o mais frequente foi a meningite. O peso e tamanho são variáveis importantes a considerar quando se avalia e tratamento de doenças infecciosas em pediátrica odontogénica população, pois podem ser factores de risco associados com o aparecimento de complicações sistémicas. Palavras-chave: celulite odontogenic facial, complicações sistêmicas, baixo peso, baixa estatura.

Palavras chave: complicações sistêmicas, celulite facial, baixo peso, baixa estatura

\section{Original article}

\section{Systemic complications derived from odontogenic facial cellulitis in Venezuelan children under the age of 6.}

\begin{abstract}
Aim: To determine the prevalence of systemic complications derived from odontogenic facial cellulitis and its relation to weight and height of children from 1 to 6 years hospitalized in the Pediatric Infectology Service of the University Hospital of Caracas in a period between 2006
\end{abstract}

and 2014. Materials and methods: An epidemiological and prevalence, non-experimental, retrospective, cross-sectional and analytical study was performed in convenience sample selected from 310 children aged 1 to 6 years. Data was analyzed by frequencies, percentages, averages, chi square test, Pearson correlation and Odds-Ratio. Results: The prevalence of systemic complications derived 
from odontogenic facial cellulitis in this sample was $32 \%$ including: meningitis $15 \%$, brain abscess $2 \%$, pneumonia $6 \%$, infective endocarditis $5 \%$ and septicemia $4 \% .65 .48 \%$ of the total sample presented low height and $68.06 \%$ low weight. Low weight was statistically related to the presence of systemic complications in this population. When compared with children of normal height and weight, children with low height (10th percentile) were 3 times more likely to present systemic complications and low weight children (10th percentile) 4 times higher chance for systemic complications.

\section{Introducción}

La celulitis facial odontogénica fue definida por Unkel J. et al. ${ }^{1}$ como una infección de la región orofacial proveniente de la dentición y de sus estructuras de soporte adyacentes. Constituye una complicación relativamente frecuente en la población pediátrica, la cual según Barrios Z. y Carrero $\mathrm{T},{ }^{2}$, deriva en un $99 \%$ de los casos de la caries dental avanzada que cursa con necrosis pulpar y lesión perirradicular.

Según la Organización Mundial de la Salud (OMS), citada por Gutiérrez S. et al., ${ }^{3}$ la celulitis es una inflamación difusa de los tejidos blandos que no está circunscrita, o limitada a una región, pero que a diferencia del absceso, tiende a diseminarse a través de los espacios tisulares y a lo largo de los planos aponeuróticos. En este sentido, Lima M., ${ }^{4}$ define a la celulitis facial odontogénica como un proceso inflamatorio agudo, sin tendencia a la limitación, que abarca varios espacios aponeuróticos de la cara y que se encuentra condicionada por dos factores: la extraordinaria virulencia del microorganismo que la produce y el estado decadente del hospedero que la padece. En relación con el portador, se le da especial atención a factores lo-
Conclusions: There are several systemic complications derived from an odontogenic facial cellulitis and can compromise the patient's life. In this study the most frequent was meningitis. Weight and height are important variables to consider when evaluating and treating odontogenic infectious diseases in the pediatric population, since may constitute risk factors in the development of associated systemic complications.

Keywords: odontogenic facial cellulitis, systemic complications, low weight, low heigth

cales como abscesos de origen endo-periodontales generalmente asociados a caries no tratadas o a tratamientos odontológicos defectuosos.

Esta patología es de origen multifactorial, sin embargo en un gran porcentaje de la población comienza por una lesión de caries, la cual es una entidad prevenible y a pesar de los esfuerzos mundiales para lograrlo, no ha sido posible. ${ }^{2,5} \mathrm{En}$ el estudio realizado por Medina J. en el año 2012, se reportó que el 78,6\% de los casos de celulitis facial odontogénica presentaron una lesión de caries avanzada como foco de infección inicial. ${ }^{6}$

Las infecciones odontogénicas son frecuentes en la población pediátrica en especial en niños menores de 6 años y la celulitis facial ha tenido un considerable aumento en su prevalencia en la última década en la población pediátrica venezolana.

En ocasiones, resulta un reto para el clínico establecer la etiología primaria de esta entidad, así como los microorganismos responsables de la misma, esto se debe a la proximidad entre la piel con los dientes, las glándulas salivales, los senos maxilares y el conducto auditivo. La celulitis facial odontogénica es similar en niños y en adultos, sin embargo, 
en el paciente pediátrico deberán tomarse en cuenta consideraciones especiales por su evolución aguda característica lo cual facilita la diseminación del proceso infeccioso a planos profundos. ${ }^{7}$

En este sentido, la celulitis facial odontogénica se produce por la extensión de un proceso infeccioso de etiología intrabucal el cual se disemina a espacios anatómicos vecinos por contigüidad o por vía hematógena, pudiendo ocasionar en ambos casos, complicaciones sistémicas como la sinusitis odontogénica, infecciones intracraneales, osteomielitis de los maxilares, tromboflebitis yugular supurada y erosión carotidea, dependiendo del área anatómica afectada en cada caso en particular, destacando la afección del mediastino y la erosión carotidea las cuales pueden ocasionar en casos extremos la muerte del paciente ya que durante su evolución, comprometen la vascularización cerebral y la permeabilidad de las vías respiratorias, en especial en individuos con estados de inmunosupresión previos a la instauración de la infección odontogénica. ${ }^{8}$

La prevalencia de la celulitis facial odontogénica en la población infantil ha ido en ascenso, en especial en aquellos países en vías de desarrollo, muestra de ello es el estudio realizado por Grandas A. y Velásquez S. ${ }^{9}$, quienes reportan una prevalencia de esta entidad de $37,45 \%$.

En Venezuela, se han implementado programas de prevención de caries y de salud bucal, sin embargo, en la actualidad se siguen reportando casos de celulitis facial odontogénica en niños de bajo nivel socioeconómico principalmente, quienes entre otras presentan deficiencias nutricionales reflejadas en una alta prevalencia de bajo peso y talla para su edad. Otros aspectos a considerar son el difícil acceso a la salud odontológica, la crisis humanitaria que engloba escasez de alimentos y medicinas y la falta de conocimiento por parte de padres, representantes y/o cuidadores de la importancia del tratamiento de la caries dental en la población pediátrica, la cual aun cuando es una entidad prevenible y tratable, su avance puede ocasionar complicaciones sistémicas y comprometer la vida de sus hijos o representados.

A nivel nacional, no se han realizado investigaciones que determinen la prevalencia de complicaciones sistémicas derivadas de celulitis facial odontogénica en niños menores de 6 años y que a su vez relacionen las mismas con el peso y la talla de dicha población.

Es de sumo interés conocer las cifras actuales de complicaciones sistémicas derivadas de esta entidad, para así establecer medidas preventivas en las poblaciones afectadas, incentivar la incorporación del odontopediatra en el trabajo transdisciplinario entre el pediatra y la madre o el cuidador, para de esta forma contribuir con el mejoramiento de la salud pública y de la calidad de vida de los niños venezolanos.

El propósito de esta investigación fue determinar la prevalencia de complicaciones sistémicas derivadas de celulitis facial odontogénica y su relación con el peso y la talla de niños de 1 a 6 años hospitalizados en el Servicio de Infectología Pediátrica del Hospital Universitario de Caracas en el periodo 2006-2014.

\section{Materiales y métodos:}

Se realizó una investigación bajo un diseño epidemiológico de prevalencia, no experimental, retrospectivo, transversal y analítico en una muestra seleccionada a conveniencia de 310 niños que cumplieron con los siguientes criterios de inclusión/exclusión: Criterios de Inclusión: niños entre 
1 y 6 años, de ambos géneros, sin antecedente de enfermedades sistémicas ni síndromes, hospitalizados en el Servicio de Infectología Pediátrica del Hospital Universitario de Caracas por diagnóstico de celulitis facial odontogénica en el periodo enero 2006 - diciembre 2014. Criterios de Exclusión: celulitis facial no odontogénica, alteraciones congénitas en el desarrollo de la dentición.

La recolección de los datos se realizó a partir del libro de control de ingresos de hospitalización y de las historias clínicas del Servicio de Infectología Pediátrica del Hospital Universitario de Caracas correspondientes al periodo enero 2006 - diciembre 2014, para ello se empleó un instrumento ad hoc el cual constó de 5 partes: datos de ingreso a la entidad hospitalaria, datos sociodemográficos del paciente, datos biológicos referentes a la entidad analizada y presencia de complicaciones sistémicas. Dicho instrumento, previo a su aplicación, fue sometido a la evaluación y aprobación de 4 expertos en el área. Los datos fueron recolectados por el investigador principal de enero a junio de 2016. Esta investigación se realizó con la autorización del Jefe del Servicio de Infectología Pediátrica del Hospital Universitario de Caracas y contó con el aval del Comité de Bioética de la Facultad de Odontología de la Universidad Central de Venezuela (CB-MG-OP-013-2015).

Todos los datos recolectados fueron introducidos en la base de datos del paquete estadístico SPSS versión 21.0 y los análisis estadísticos se realizaron con un intervalo de confianza del 95\% y un nivel de significancia p-valor 0,05.

Se realizó un análisis descriptivo con el fin de caracterizar la muestra basado en frecuencias, porcentajes, promedios y desviaciones estándar. Igualmente, se realizó un análisis epidemiológico, con la finalidad de determinar la prevalencia de complicaciones sistémicas derivadas de la celulitis facial odontogénica en la población estudiada, basado en la prueba estadística de chi cuadrado de independencia entre variables y un análisis comparativo, analítico y correlacional entre las variables peso, talla y complicaciones sistémicas utilizando la prueba estadística de correlación de Pearson y la prueba de riesgo Odds-Ratio.

\section{Resultados:}

Al identificar la presencia de complicaciones sistémicas en la muestra analizada, se encontró que de un total de 310 niños que cumplieron con los criterios de selección, 99 exhibieron complicaciones sistémicas derivadas de celulitis facial odontogénica, resultado que determina una prevalencia del $32 \%$. Al aplicar la prueba estadística de chi cuadrado para evaluar si existía relación estadísticamente significativa entre la celulitis facial odontogénica y la presencia de complicaciones sistémicas se obtuvo un valor de significancia $\mathrm{p}=0,025$ ( $\mathrm{p}$-valor $<0,05$ ) y una asociación entre ambas variables $r=22,4 \%$, resultado que indica una relación estadísticamente significativa entre ambas variables en los niños que conformaron esta muestra poblacional. En la Tabla 1, se aprecian estos resultados.

En esta investigación se reportan 5 complicaciones sistémicas derivadas de celulitis facial odontogénica en la población estudiada: meningitis, absceso cerebral, endocarditis infecciosa, neumonía y septicemia. La meningitis fue encontrada en 46 pacientes pediátricos, resultado que representa una prevalencia del 15\% de esta entidad. Al aplicar el estadístico de chi cuadrado de Pearson se determinó una relación entre ambas variables de $\mathrm{r}=17,1 \%$ y una significancia de $\mathrm{p}=0,045$ ( $\mathrm{p}$-valor $<0,05)$, indicando que en esta muestra, la celulitis facial odontogénica se asoció estadísticamente con 


\begin{tabular}{|c|c|c|c|c|c|c|}
\hline & $\begin{array}{c}\text { Complicaciones } \\
\text { Sistémicas }\end{array}$ & Meningitis & $\begin{array}{l}\text { Absceso } \\
\text { Cerebral }\end{array}$ & $\begin{array}{l}\text { Endocarditis } \\
\text { Infecciosa }\end{array}$ & Neumonía & Septicemia \\
\hline Presencia & 99 & 46 & 7 & 16 & 17 & 13 \\
\hline Ausencia & 211 & 264 & 303 & 294 & 293 & 297 \\
\hline Prevalencia & $32 \%$ & $15 \%$ & $2 \%$ & $5 \%$ & $6 \%$ & $4 \%$ \\
\hline $\mathbf{X}^{2}$ & 5,0138 & 3,8829 & 1,0846 & 1,3425 & 2,9549 & 1,6288 \\
\hline gl & 1 & 1 & 1 & 1 & 1 & 1 \\
\hline $\mathbf{p}$ & 0,025 & 0,045 & 0,302 & 0,201 & 0,198 & 0,265 \\
\hline $\mathbf{r}$ & $.224^{\star \star}$ & $.171^{\star}$ & .015 & .035 & .052 & .018 \\
\hline
\end{tabular}

Chi Cuadrado de Pearson (X2). Grados de libertad (gl). Significancia Estadística (p): Sig.<0,05 (95\%). En la Tabla se resalta en azul la significancia $<0,05$. Correlación de Pearson $(r):{ }^{\star}$. La correlación es significante al nivel 0,05 (bilateral). ${ }^{\star *}$. La correlación es significativa al nivel 0,01 (bilateral).

Tabla 1. Complicaciones sistémicas derivadas de la celulitis facial odontogénica en la población estudiada.

la presencia de meningitis. Dichos hallazgos se muestran en la Tabla 1.

El absceso cerebral se observó en 7 individuos, la endocarditis infecciosa en 16 pacientes, la neumonía en 17 niños y la septicemia en 13, lo que indica una prevalencia del $2 \%, 5 \%, 6 \%$ y $4 \%$ respectiva-

\begin{tabular}{|c|c|c|}
\hline Talla (Percentiles) & Frecuencia & Porcentaje (\%) \\
\hline $\mathbf{P}_{10}=$ Baja & 203 & $\mathbf{6 5 , 4 8}$ \\
\hline $\mathbf{P}_{50}=$ Normal & 107 & 34,51 \\
\hline $\mathbf{P}_{90}=$ Alta & 0 & 0 \\
\hline Total & $\mathbf{3 1 0}$ & $\mathbf{1 0 0}$ \\
\hline Peso (Percentiles) & Frecuencia & Porcentaje (\%) \\
\hline $\mathbf{P}_{10}=$ Bajo & 211 & $\mathbf{6 8 , 0 6}$ \\
\hline $\mathbf{P}_{50}=$ Normal & 99 & 31,93 \\
\hline $\mathbf{P}_{90}=$ Alto & 0 & 0 \\
\hline Total & 310 & 100 \\
\hline
\end{tabular}

Tabla 2. Distribución de la muestra de acuerdo a la talla y el peso expresados en percentiles. mente. Al emplear la prueba de chi cuadrado no se encontró significancia a nivel estadístico en esta muestra: $\mathrm{p}=0,302$ para el absceso cerebral, $\mathrm{p}=0,201$ para la endocarditis infecciosa, $\mathrm{p}=0,198$ para la neumonía y $\mathrm{p}=0,265$ para la septicemia ( $\mathrm{p}$-valor $>0,05$ ), indicando que en esta población, estas complicaciones sistémicas no presentaron relación estadística con la presencia de celulitis facial odontogénica. En la Tabla 1 se presentan estos resultados.

En cuanto al peso y la talla de los pacientes con celulitis facial odontogénica pertenecientes a la muestra analizada, ambas variables fueron valoradas en kilogramos (kgs) y centímetros (cms) respectivamente y medidas a partir de percentiles, a saber percentil 10 (bajo), percentil 50 (normal) y percentil 90 (alto), empleando como valores de referencia los propuestos por FUNDACREDESA ${ }^{10}$ para niños venezolanos.

En la Tabla 2 se aprecia la distribución de la población de acuerdo a la talla expresada en percentiles y en la misma se observa que el 65,48\% se ubicó en el percentil 10, el promedio de talla fue de 93,50 
cms, con una desviación estándar de $\pm 10,7$ cms, el valor mínimo registrado fue de $69,5 \mathrm{cms}$ y el máximo de 113,3 cms.

Con respecto a la distribución de la población de acuerdo al peso el mismo está expresado en percentiles y en esta investigación se observó que el 68,06\% de los niños se ubicaron dentro del percentil $10 \mathrm{y}$ el promedio de peso fue de $12,97 \mathrm{kgs}$ con una desviación estándar de $\pm 2,52$ $\mathrm{kgs}$, el valor mínimo registrado fue de $7,6 \mathrm{kgs}$ y el máximo de 19,4 kgs. Estos resultados se aprecian en la Tabla 2.

Al relacionar la presencia de complicaciones sistémicas con el peso y la talla de la población estudiada, se encontró luego de aplicar la prueba estadística de correlación de Pearson, una relación estadísticamente significativa con el peso ( $\mathrm{p}=0,048 \mathrm{p}$-valor $<0,05 \mathrm{y}$ una correlación de $\mathrm{r}=20,7 \%$ ), resultado que indica que el peso del paciente se puede relacionar con la presencia de complicaciones sistémicas derivadas de celulitis facial odontogénica, sin embargo, para la talla no se halló significancia estadística $(p=0,207$ $\mathrm{p}$-valor $>0,05)$. A continuación, en la Tabla 3 se presenta la prueba estadística empleada.

\begin{tabular}{|c|c|c|}
\cline { 2 - 3 } \multicolumn{1}{c|}{} & Peso $(\mathrm{Kgs})$ & Talla $(\mathrm{cms})$ \\
\hline $\begin{array}{c}\text { Complicaciones } \\
\text { Sistémicas }\end{array}$ & 99 & 99 \\
\hline$p$ & 0,048 & 0,207 \\
\hline $\mathrm{r}$ & $.207^{*}$ & .134 \\
\hline
\end{tabular}

Significancia Estadística (p): Sig. $<0,05$ (95\%). En la Tabla se resalta en azul la significancia $<0,05$.

CorrelacióndePearson(r): ${ }^{*}$.Lacorrelaciónessignificantealnivel0,05 (bilateral). ${ }^{\star *}$. La correlación es significativa al nivel 0,01 (bilateral).

Tabla 3. Correlación de Pearson para las variables complicaciones sistémicas, peso y talla.
Por su parte, en este estudio se quiso evaluar la probabilidad de presentar o no complicaciones sistémicas derivadas de una celulitis facial odontogénica en niños con percentil normal y bajo con respecto a las variables antropométricas talla y peso. Para ello se realizó el análisis de Odds Ratio. En este sentido, al analizar la talla, aquellos pacientes que estaban dentro del percentil 10 (baja talla), exhibieron 3 veces más probabilidades de presentar complicaciones sistémicas al compararlos con los niños cuya talla se ubicó dentro del percentil 50 (talla normal). Seguidamente, en la Tabla 4, la prueba estadística empleada.

Con respecto al peso, los niños con bajo peso, es decir, que estaban dentro del percentil 10, exhibieron 4 veces más probabilidades de presentar complicaciones sistémicas al compararlos con niños cuyo peso se agrupó en el percentil 50. A continuación, en la Tabla 5, se presenta la prueba estadística.

\section{Discusión.}

En esta investigación se reportan cinco complicaciones sistémicas derivadas de la celulitis facial odontogénica cuya etiología principal fue la caries dental: meningitis, neumonía, endocarditis infecciosa, septicemia y absceso cerebral. Grandas A. y Velásquez S., ${ }^{9}$ contrariamente a este estudio, no encontraron complicaciones sistémicas en un $97,86 \%$ de los pacientes estudiados. No obstante, es importante puntualizar que en el Servicio de Infectología Pediátrica del Hospital Universitario de Caracas se hospitalizan aquellos pacientes que no responden de forma adecuada al tratamiento pediátrico instaurado previamente, siendo referidos a este servicio especializado, por lo que ya ingresan con ciertas complicaciones de base. 


\begin{tabular}{|c|c|c|c|c|c|}
\hline \multicolumn{6}{|c|}{ Recuento } \\
\hline & & & \multicolumn{2}{|c|}{ Complicaciones Sistémicas } & \multirow[t]{2}{*}{ Total } \\
\hline & & & SI & NO & \\
\hline \multirow[t]{4}{*}{ Talla } & $\mathrm{P} 10$ & $\mathrm{~N}$ & 79 & 124 & 203 \\
\hline & & $\begin{array}{l}\text { \% Complicaciones } \\
\text { Sistémicas }\end{array}$ & $79,7 \%$ & $58,7 \%$ & $65,5 \%$ \\
\hline & $\mathrm{P} 50$ & $\mathrm{~N}$ & 20 & 87 & 107 \\
\hline & & $\begin{array}{c}\text { \% Complicaciones } \\
\text { Sistémicas }\end{array}$ & $19,8 \%$ & $41,2 \%$ & $34,5 \%$ \\
\hline \multirow[t]{2}{*}{ Total } & & $\mathrm{N}$ & 99 & 211 & 310 \\
\hline & & $\begin{array}{c}\text { \% Complicaciones } \\
\text { Sistémicas }\end{array}$ & $100,0 \%$ & $100,0 \%$ & $100,0 \%$ \\
\hline
\end{tabular}

\begin{tabular}{|cccc|}
\hline \multicolumn{3}{|c|}{ Riesgo Estimado } & \\
\hline & Valor & \multicolumn{2}{c|}{ 95\% Intervalo de confianza } \\
& & Inferior & Superior \\
\hline Odds Ratio para Talla (P10, P50) & 2,7713 & W1,5798 & 4,8313 \\
\hline Presencia de complicaciones sistémicas & 2,082 & 1,3909 & 3,1643 \\
\hline Ausencia de complicaciones sistémicas & 2,4207 & 1,6172 & 3,6234 \\
\hline N de casos válidos & 310 & & \\
\hline
\end{tabular}

Tabla 4. Análisis Odds-Ratio para complicaciones sistémicas y talla según el percentil.

La meningitis fue la complicación sistémica más frecuente en este estudio y la misma presenta una tasa de mortalidad que alcanza un $4,5 \%$ en los países desarrollados en niños menores de 10 años, otras consecuencias que se pueden derivar de esta complicación son: a nivel cardiovascular, la sepsis y a nivel neurológico, las convulsiones o parálisis en pares craneales. En aproximadamente una cuarta parte de los niños afectados, la meningitis puede dejar secuelas permanentes tan graves como la epilepsia, el retraso psicomotor e incluso sordera neurosensorial (la cual es la más frecuente). ${ }^{11}$

Con respecto a la neumonía, en la literatura se citan como consecuencias derivadas de esta entidad, el derrame pleural, la neumonía necrotizante y en menor medida la sepsis. ${ }^{12}$ Por su parte, en lo referente a las complicaciones por la endocarditis infecciosa, las más comunes son la insuficiencia cardiaca congestiva ( $50 \%$ de los niños), complicaciones embólicas (20\% de los niños) que desencadenan alteraciones neurológicas, abscesos cerebrales y hemorragias, embolismo pulmonar, soplo (90\% de los pacientes), esplenomegalia (55-70\%) y derrame pleural con menor periodicidad. ${ }^{13}$

Otra complicación encontrada en este estudio fue el absceso cerebral el cual presenta como consecuencias la muerte en el 10\% de los casos pediátricos, los pacientes pueden quedar con secuelas neurológicas como daño neurológico, disartria o paresia leve, frontalización, hemiparesia, fístula de líquido cefalorraquídeo y alteración transitoria de la memoria. ${ }^{14}$ La septicemia también es otra complicación hallada en esta población. Esta enti- 


\begin{tabular}{|c|c|c|c|c|c|}
\hline \multicolumn{6}{|c|}{ Recuento } \\
\hline & & & \multicolumn{2}{|c|}{ Complicaciones Sistémicas } & \multirow[t]{2}{*}{ Total } \\
\hline & & & SI & NO & \\
\hline \multirow[t]{4}{*}{ Peso } & $\mathrm{P} 10$ & $\mathrm{~N}$ & 83 & 128 & 211 \\
\hline & & $\begin{array}{c}\% \text { Complica- } \\
\text { ciones Sistémicas }\end{array}$ & $83,8 \%$ & $60,6 \%$ & $68,1 \%$ \\
\hline & P50 & $\mathrm{N}$ & 16 & 83 & 99 \\
\hline & & $\begin{array}{c}\text { \% Complica- } \\
\text { ciones Sistémicas }\end{array}$ & $16,1 \%$ & $39,3 \%$ & $31,9 \%$ \\
\hline \multirow[t]{2}{*}{ Total } & & $\mathrm{N}$ & 99 & 211 & 310 \\
\hline & & $\begin{array}{c}\% \text { Complica- } \\
\text { ciones Sistémicas }\end{array}$ & $100,0 \%$ & $100,0 \%$ & $100,0 \%$ \\
\hline
\end{tabular}

\begin{tabular}{|cccc|}
\hline & Riesgo Estimado & \\
\hline & Valor & & 95\% Intervalo de confianza \\
\hline Odds Ratio para Peso (P10, P50) & 3,5005 & Inferior & Superior \\
\hline Presencia de complicaciones sistémicas & 2,4339 & 1,9169 & 6,3922 \\
\hline Ausencia de complicaciones sistémicas & 0,7223 & 1,5077 & 3,9289 \\
\hline N de casos válidos & 310 & 0,4474 & 1,1659 \\
\hline
\end{tabular}

Tabla 5. Análisis Odds-Ratio para complicaciones sistémicas y peso según el percentil.

dad se considera como la novena causa de muerte en niños entre 1 y 4 años de edad, con una mortalidad anual estimada de 0,5 por cada 100.000 niños. Otra secuela puede ser un shock séptico. ${ }^{15}$

En este estudio se consideraron variables antropométicas como el peso y la talla a fin de evaluar el estado nutricional de los niños hospitalizados con diagnóstico de celulitis facial odontogénica y su relación con la presencia de complicaciones sistémicas, esto debido a que de los 310 pacientes que representaron la población de estudio, se encontró que 203 niños estaban dentro del percentil 10 (bajo) para la talla y 211 para el peso de acuerdo a los valores de referencia de la población venezolana suministrados por FUNDACREDESA. ${ }^{10}$ Este hallazgo hace reflexionar sobre la importancia de considerar el peso y la talla a la hora de evaluar enfermedades infecciosas como la celulitis facial odontogénica en la población pediátrica venezolana, ya que al determinar la probabilidad de presentar o no complicaciones sistémicas en individuos con percentil normal o bajo se obtuvo que en esta muestra, los niños con percentil bajo para la talla tenían 3 veces más probabilidad de presentar complicaciones sistémicas en comparación con los niños con percentil normal. Por su parte, aquellos pacientes con percentil bajo para el peso presentaron 4 veces más probabilidad de complicaciones sistémicas que los niños con percentil normal, hallazgo que indica que la baja talla y el bajo peso pueden constituir un factor de riesgo para las complicaciones sistémicas derivadas de celulitis facial odontogénica en niños entre 1 y 6 años de edad.

En la literatura no se encontraron estudios que 
consideraran el peso y la talla de la población estudiada y lo relacionaran con la presencia de complicaciones sistémicas derivadas de una celulitis facial odontogénica en niños menores de 6 años. Resulta relevante destacar la importancia que tiene el conocimiento por parte del odontopediatra y del pediatra del percentil de peso y de la talla de cada paciente ya que ambos pueden constituir factores de riesgo en la evolución de enfermedades infecciosas de origen bucal, las cuales en ocasiones pueden derivar en complicaciones sistémicas que comprometan la calidad de vida y el estado de salud de la población pediátrica.

\section{Conclusiones.}

Las complicaciones sistémicas que se pueden derivar de una celulitis facial odontogénica son varias, en este estudio se encontraron: meningitis la cual fue la más prevalente en el $15 \%$ de la muestra, neumonía en el $6 \%$, endocarditis infecciosa en el $5 \%$, septicemia en el $4 \%$ y absceso cerebral en el $2 \%$. Es importante señalar que todas estas afecciones pueden poner en riesgo la vida del paciente y son consecuencia de la caries, la cual es una entidad prevenible y tratable, de allí la importancia del tratamiento odontopediátrico acertado y oportuno.

El peso y la talla son variables antropométricas que deben ser consideradas a la hora de evaluar y tratar enfermedades infecciosas odontogénicas como la celulitis facial, ya que el bajo peso y la baja talla pueden constituir factores de riesgo a la aparición de complicaciones sistémicas.

\section{Referencias bibliográficas}

1. Unkel J, Mckibben D, Fenton S, Nazif M, Moursi A, Schuit K. Comparison of odontogenic and non odontogenic facial cellulitis in a pediatric hospital population. Pediatric Dent. 1997; 19: 476-9.

2. Barrios Z, Carrero T. Prevalencia de infecciones de origen pulpar en los niños atendidos en el Servicio de Odontopediatría del Instituto Autónomo Hospital Universitario de los Andes, Mérida, Venezuela. Rev. Odont. los Andes. 2011; 6: $42-51$.

3. Domínguez S, Machín A, Paredes M, Rosete M. Comportamiento de la celulitis cervico-facial en una comunidad venezolana. Rev Ciencias Médicas. 2012;16(2).

4. Lima M. Impacto social de la estrategia para disminuir la incidencia de celulitis facial odontógena. Rev Med Electrón. 2009 ; 31.

5. Guerra M, Hernández M, Carrasco W, Albornoz E. Intervención odontosanitaria para controlar caries en dentición primaria en hijos de madres VIH/SIDA. Acta Odontológica Venezolana. 2014; 52.

6. Medina J. Celulitis facial odontogénica en pacientes hospitalizados en un hospital especializado en pediatría. Dermatol Perú. 2012; 23: 73-7.

7. Cores A, Chaviano J, Mazaira L, Atienza L, Salas M. Tratamiento y evolución de la celulitis facial odontógena en edad pediátrica. Gaceta Dental. 2008; 221: 162-181.

8. Gutierrez J, Perea E, Romero M, Girón J. Infecciones orofaciales de origen odontogénico. Med Oral Patol Oral Cir Bucal. $2004 ; 9$.

9. Grandas A, Velásquez S. Prevalencia de celulitis odontogénica en pacientes de 0-18 años que asistieron a la Fundación HOMIHospital de la Misericordida de Bogotá entre febrero de 2009- febrero 2011. Acta Odontológica Colombiana. 2012 ; 2: 71-85.

10. FUNDACREDESA. Tabla de Peso, Talla, Circunferencia Cefálica y Circunferencia de Brazo de las Venezolanas y

Venezolanos. 1994. 
11. Baquero F, Vecino R, del Castillo F. Meningitis Bacteriana. Protocolos diagnóstico-terapéuticos de la AEP: Infectología Pediátrica. Madrid; 2012.

12. Aracil F. Mesa Redonda: Infección neumocócica desde distintas perspectivas. Bol Pediatr. 2008; 48: 183-8.

13. Oliviera C, Lubián S. Endocarditis Infecciosa. Tratamiento y Profilaxis. Protocolos Diagnósticos y Terapéuticos en Cardiología Pediátrica. Madrid; 2005.

14. Fica A, Bustos P, Miranda G. Absceso cerebral. A propósito de una serie de 30 casos. Rev Chil Infect. 2006; 23 : 140-9.

15. Paganini H. Tratamiento de la sepsis en pediatría: ¿Qué debemos hacer? Arch Argent Pediatr. 2003; 101: 406-6.

Recibido: 30/05/2017

Aceptado: 28/02/2018

Correspondencia: Gabriel Alejandro Zambrano. gazblondell@gmail.com. 